\title{
The SED Machine - Fast classification of transient objects
}

\author{
Andreas Ritter ${ }^{1}$, N. Konidaris ${ }^{2}$, C. C. Ngeow $^{1}$, R. Quimby ${ }^{3}$ \\ and S. Ben-Ami ${ }^{4}$ \\ ${ }^{1}$ Graduate Department of Astronomy, National Central University, \\ email: azuri.ritter@gmail.com \\ ${ }^{2}$ California Institute of Technology, CA, USA ${ }^{3}$ University of Tokyo, Japan ${ }^{4}$ The Weizmann \\ Institute of Science, Israel
}

\begin{abstract}
The field of time-domain astronomy is expected to enjoy a golden age during this decade. However, the traditional method for the classification of transient candidates using spectra obtained with medium- to large-aperture telescopes is extremely time consuming and struggling to keep up with the discovery rate. The Spectral Energy Distribution (SED) Machine uses a new approach in order to overcome this shortcoming.
\end{abstract}

Keywords. instrumentation: spectrographs, techniques: photometric, techniques: spectroscopic, telescopes, stars: novae, gamma rays: bursts

The Palomar Transient Factory (PTF, Law 2009, Rau 2009) is currently discovering $\sim 4,000$ candidate transients per year. Traditionally these transient candidates are classified spectroscopically using medium to large aperture telescopes. Even with a relatively large amount of telescope time, only about $10 \%$ of the transient candidates can be followed-up and classified. In order to overcome this shortcoming for PTF and other future large-scale transient surveys, a new approach is needed.

The SED Machine (Ben-Ami 2012) was specifically designed to classify transients at a high rate using small-aperture $(1-2 \mathrm{~m})$ telescopes. Spectra taken at a resolution of $\mathrm{R}$ $\sim 100$ contain as much information for classification as $\mathrm{R} \sim 1,000$ spectra, but with an increase in signal-to-noise per second of a factor of 3. This means that the SED Machine is sufficient to classify transients at a high success rate using small-aperture telescopes.

The SED Machine hosts a 4-colour Rainbow Camera (RC) for target acquisition and flux calibration, as well as the IFU for the spectroscopic analysis of the transient candidates. The IFU utilises a triple prism for a nearly constant resolution of about $\mathrm{R} \sim 100$ in order to efficiently classifying the transient object. 3,600 hexagonal lenslets, each covering $\sim 0.675^{\prime \prime}$ on the sky, deliver spectra of the target, the host galaxy, and the sky background. The RC is a photometric subsystem taking images at 4 bands simultaneously: Sloan $u, g, r$, and $i$. The comparison of the reduced photometric images to high-quality photometric catalogues will allow for the correction of the simultaneously acquired spectra for atmospheric extinction, seeing variations, and other time dependent factors that affect the system transmittance.

The Data-Reduction Pipeline (DRP) for the IFU is based on the STELLA DRP (Ritter \& Washuettl, 2004). It includes the standard data-reduction steps such as bad-pixel correction, overscan and Bias subtraction, Flat-fielding, and wavelength calibration. New instrument-specific procedures have been developed for spectra tracing, scattered-light subtraction, optimal extraction (Ritter et al., subm.), and sky subtraction of the spectra (Ritter et al., in prep.). 